\title{
An audit on medical students' exposures to occupational hazards during cadaveric dissections
}

Mathangasinghe $\mathrm{Y}^{1}$, Samaranayake UMJE${ }^{1}$, Perera MHS ${ }^{1}$, Maddumaarachchi HSTM ${ }^{1}$, Anthony $\mathrm{DJ}^{1}$, Malalasekera $\mathrm{AP}^{1}$

${ }^{I}$ Department of Anatomy, Faculty of Medicine, University of Colombo, Colombo, Sri Lanka ${ }^{\prime}$ Faculty of Medicine, University of Peradeniya, Sri Lanka

\begin{abstract}
\section{Introduction}

We intended to study the prevalence of occupational hazards in the anatomy laboratory and the safety measures taken by students.
\end{abstract}

\section{Methodology}

An audit was conducted among two batches of medical students, soon after the completion of the anatomy curriculum. Exposures, practices, health conditions of the students, their suggestions to improve safety measures and self-reported engagement in dissections in each session (in a ten-point Likert scale) were assessed using a validated self-administered questionnaire.

\section{Results}

Of 196 respondents, $50 \%(\mathrm{n}=98)$ were males. Mean age was $21.8 \pm 1.1$ years. During their first-year dissections, $15.8 \%(n=31)$ had cut injuries, $86.7 \%(n=170)$ had skin contact with cadaver fluids while $5.6 \%(n=11)$ had eye splashes. Of those with skin contact, $43.5 \%(n=74)$ washed immediately. Of those who had eye splashes, only
$18.2 \%(n=2)$ washed immediately for 20 minutes as recommended. Nine students reported new nail infections, seven developed allergies to cadaver fluids and 15 developed skin rashes during the period of dissections. Self-reported engagement in dissections positively correlated with the frequency of skin contact with cadaver fluids $(r=.161, p=0.035)$, but not with the frequency of cut injuries $(\mathrm{p}>.05)$ or eye splashes ( $p>.05)$. Students suggested the following safety measures: $63.3 \%(\mathrm{n}=72)-1 \mathrm{lab}$ coats, $55.6 \%(\mathrm{n}=109)$ masks, $16.8 \%(n=33)$-g og gles, $43.9 \%(n=86)$-a lecture on lab safety, $44.4 \%(n=87)-a$ workshop and $40.3 \%(\mathrm{n}=79)$-a formal safety protocol.

\section{Conclusions}

Students are exposed to numerous occupational hazards in the anatomy laboratory. Inadequate adherence to internationally recommended laboratory safety measures may increase the risk of occupational hazards.

\section{Keywords}

anatomy, laboratory, health hazards, occupational exposure, formalin 
Mathangasinghe Y, Samaranayake UMJE, Perera MHS, Maddumaarachchi HSTM, Anthony DJ, Malalasekera AP - An audit on medical students' exposures to occupational hazards during cadaveric dissections

\section{Introduction}

Cadaveric dissections play an integral role in various medical disciplines [1-3]. Dissections do not merely improve the theoretical knowledge, but also improve students' manual dexterity, threedimensional comprehension, professional behaviour and create a humane concept of life and death [3]. But this does not come without risks. Fresh cadavers act as an excellent culture medium for many pathogens including bacteria (Mycobacterium tuberculosis, Salmonella typhi, Brucella spp), viruses (hepatitis B, C and Human Immunodeficiency Virus), fungi and prions [1, 4, 5]. Some studies have identified the presence of pathogenic agents even in formalin preserved cadavers after many months of preservation $[1,2]$. The efficacy of disinfectants used during fixation of cadavers are not well understood [6]. Hence, the method of preservation and the age of the cadavers may create a "false sense of security" [5]. Therefore, every cadaver should be considered as an infectious material $[1,4-6]$.

Various chemicals used in dissections such as fixatives, disinfectants and buffers pose potentially harmful health effects $[1-3,5,6]$. Some of these agents may be flammable, explosive, acidic or carcinogenic [5]. Formalin is one such preservative which is flammable and classified as a "probable human carcinogen" [7, 8]. Carcinogenic properties of most of the chemicals used in cadaveric preservations are largely unknown [6].

The students and ancillary staff are the most involved with dissections, therefore amenable to health risks [1-3, 5]. Studies demonstrate a substantial lack of awareness on health hazards among students $[1,3,5]$ and the awareness is significantly lower among the participants in anatomy laboratories when compared to other laboratories which handle cadavers [2]. Establishment of a healthy environment within the laboratory with safety measures and proper codes of conduct have shown to minimize potential injuries to the involved individuals $[1,2$, 5].

Safety protocols for laboratories conducting cadaveric studies vary widely among different communities [5]. At the time of designing this study, there were no such protocols in many leading Universities in Sri Lanka. To develop protocols, it is essential to understand the existing practice, encountered occupational hazards and the safety procedures followed by the students. Hence, the purpose of this audit was to identify the potential occupational hazards encountered by the medical students during their cadaveric dissections and to find out their practices following such incidents.

\section{Methods}

An audit was conducted among two batches of medical students of the Faculty of Medicine, University of Colombo, Sri Lanka. They were recruited for the study soon after the completion of their anatomy curriculum. The study was carried out from August to September 2018. The audit conformed to the guidelines of the Declaration of 
Mathangasinghe Y, Samaranayake UMJE, Perera MHS, Maddumaarachchi HSTM, Anthony DJ, Malalasekera AP - An audit on medical students' exposures to occupational hazards during cadaveric dissections

Helsinki [9]. All the students were invited for the study. Written informed consent was taken prior to data collection from the participants. Exposures, practices, prevention methods, health conditions of the students, their suggestions to improve safety measures and self-reported engagement in dissections during each session were assessed using a self-administered questionnaire. Exposure was tested using 'yes' or 'no' responses, while practices and safety measures were tested by selection of a single response out of a list. Students themselves reported how frequently they were involved in the dissections during the allocated time for practical sessions (self-reported engagement). Frequency of engagement in dissections was measured using a ten-point Likert scale from 1 to 10 ( $1=$ never, $10=$ always $)$. A section on ways to improve the current practice was included. The face validation was obtained through a pilot test. Data was analysed using SPSS version 20. Standard descriptive statistics were used for the analysis of the gathered data at a priori alpha of .05

\section{Results}

In this study, a total of 196 students were assessed. Male to female ratio was 1:1. Mean age was $21.8 \pm 1.1$ years.

\section{Reportedinjuries}

During their first-year dissections, 31 $(15.8 \%)$ had at least one cut injury with a sharp object (scalpel or a sharp body part such as the cut end of a rib). Following scalpel cut injuries only 7 (22.6\%) received post exposure tetanus prophylaxis. Majority $(\mathrm{n}=170,86.7 \%)$ had skin contact with cadaver fluids at least once during dissections. The mean frequency of a medical student having skin contact with cadaver fluid during a period of one year was $9 \pm 7$. Of those with skin contact, only 74 (43.5\%) washed immediately. Ninety-two $(54.1 \%)$ waited until the end of the dissection session to wash the area of skin that was in contact with cadaver fluids. A total of 11 (5.6\%) students had eye splashes with cadaver fluids. Of those who had eye splashes, only two $(18.2 \%)$ washed immediately for 20 minutes as per the recommendations $[10$, 11]. Eight (72.7\%) students washed eyes immediately with running water, however for a short duration (less than 20 minutes) following splashes. One student $(9.1 \%)$ waited until the end of the dissection to wash eyes following a minor splash.

\section{Reported health conditions}

Nine (4.6\%) students developed new nail infections during the first year. Seven (3.6\%) students developed allergies to cadaver fluids. None of them reported anaphylaxis or severe allergies necessitating hospital admissions. Fifteen $(7.7 \%)$ developed skin rashes during the period of dissections. Majority of these skin rashes developed in the areas that were more prone to come in to contact with cadaver fluids such as exposed areas of the hands and the legs. 
Mathangasinghe Y, Samaranayake UMJE, Perera MHS, Maddumaarachchi HSTM, Anthony DJ, Malalasekera AP - An audit on medical students' exposures to occupational hazards during cadaveric dissections

Students' practices during dissections

Mean score of the self-reported engagement in dissections was $7 / 10$ $(\mathrm{SD}=3 / 10)$. Self-reported mean frequency of disposing scalpels outside the sharp bin was $3 / 10(\mathrm{SD}=3 / 10)$. One student stated that he/she has never seen the sharp bin during the whole first year. Everyone in the study population had touched the cadavers without wearing gloves at least once during their first year. Three $(1.5 \%)$ students stated that they were unaware that cadaver fluid was harmful. A Pearson's product-moment correlation coefficient test was conducted to investigate the relationship between the self-reported engagement in dissections and the frequency of getting injuries in the laboratory. Preliminary analyses were conducted to ensure no violations of the assumptions of normality, linearity and homoscedasticity. There was a weak, positive correlation between the selfreported engagement in dissections and the frequency of skin contact with cadaver fluids $(\mathrm{r}=.161, \mathrm{n}=196, \mathrm{p}=0.035)$ (Figure 1). But no such correlations were identified between the engagement in dissections and the frequency of cut injuries $(p>.05)$ or frequency of eye splashes ( $p>.05)$. Independent sample ttests did not show significant differences of having cut injuries, cadaver fluid contacts with the skin or eye splashes between males and females ( $p>.05)$.

Suggestions by the students

Majority of the students ( $\mathrm{n}=72,63.3 \%)$ suggested lab coats to be worn compulsorily in the dissection hall. This was followed by the suggestion of the safety measures: wearing face masks ( $\mathrm{n}=109,55.6 \%$ ), a workshop to improve awareness of occupational hazards $(\mathrm{n}=87,44.4 \%)$, a lecture on lab safety ( $\mathrm{n}=86,43.9 \%)$, a formal safety protocol to be put up as a poster in the dissection hall $(\mathrm{n}=79,40.3 \%)$ and wearing eye protective goggles $(n=33,16.8 \%)$. Further, they requested a proper training on and adequate facilities for handwashing during the first year.

\section{Discussion}

At an anatomy laboratory a handler gets exposed to hazardous material through direct contact, inhalation or by contact with the infected instruments [12]. Biosafety is the practice used to contain infectious organisms in the laboratory environment and minimize the risk of exposure to laboratory handlers $[6,13]$. Biosafety in microbiological and biomedical laboratories (BMBL) in the United States defines biosafety level two as handling the cell cultures of humans and other primates, which include anatomy laboratories [6].

All wounds apart from the clean minor wounds should be considered tetanus prone and must follow tetanus prophylaxis [14]. Level two laboratory safety guidelines recommend immediate washing of the injured areas with running water followed by covering of the region with a bandage or sticking plaster to avoid any direct contamination of the wound $[15,16]$. In our study, of those who sustained cut injuries, 24 $(77.3 \%)$ did not take the tetanus 
Mathangasinghe Y, Samaranayake UMJE, Perera MHS, Maddumaarachchi HSTM, Anthony DJ, Malalasekera AP - An audit on medical students' exposures to occupational hazards during cadaveric dissections

vaccination. Furthermore, of those with skin contact with cadaver fluid, only 74 (43.5\%) washed immediately, while 92 (54.1\%) waited until the end of the dissection session to wash. Only two $(18.2 \%)$ students washed their eyes soon after the eye splash of cadaver fluid occurred.

According to the World Health Organization safety protocols interaction with bodily fluids of another individual is considered a health risk [13]. If splashes occur, eyes should be washed with running water or $0.9 \%$ normal saline as soon as possible for at least 20 minutes while avoiding soap or any other irritant liquids $[10,11,13]$. This is the single most important step in preventing eye injuries and spread of infections [17]. In the present study, majority of the students neglected the duration of washing of the contaminated eye. Protective eyewear was used in most biosafety level laboratories which our laboratory did not follow [12]. Nevertheless, use of protective eyewear is not seen in dissection laboratories in Sri Lankan Universities. Although wearing gloves was a policy in the anatomy laboratory, everyone in the study populations has touched the cadavers at least once during their course without wearing gloves. Direct contact with cadaveric matter is strictly advised against in many biosafety level two protocols around the world due to presence of potentially infectious organisms on the cadavers $[12,16,18]$. Several students reported skin rashes and allergies. This could be due to the lack of protective wear preventing direct contact with the cadaveric matter. Educating the students about proper hand washing techniques would help minimize the incidence of fungal infections of the nails and skin rashes. Lab coats were recommended in guidelines when conducting procedures with a potential for splashes $[13,18]$. Lab coats were not made mandatory for our students due to unavailability of air-conditioned dissection halls and the heat in the laboratory complex. However, during our study, majority of the participants were willing to wear lab coats during dissections.

According to our findings, the active involvement in cadaveric dissections was not a major determinant of the frequency of cut injuries or eye splashes. Therefore, everyone stepping into the dissection hall will be at risk of contact with occupational hazards and avoiding contact with cadavers alone will not prevent the students from getting exposed.

One student in our study was not aware of the location of the sharp object disposal bin. This highlights the need to orient the students about the proper disposal techniques and where the relevant stations or protective equipment are in the laboratory complex.

It is mandatory for the medical students to be aware of the potential hazards in anatomy laboratories and what measures need to be taken in the event of an exposure. Students should be informed and emphasized on the risks of occupational hazards in an anatomy laboratory and what protocols to follow in an event of exposure $[1,5,18]$. 
Mathangasinghe Y, Samaranayake UMJE, Perera MHS, Maddumaarachchi HSTM, Anthony DJ, Malalasekera AP - An audit on medical students' exposures to occupational hazards during cadaveric dissections

\section{Strengths and limitations of the study}

This was a single centre study. We only looked at the occupational hazards encountered by first year medical students in the anatomy laboratory. We used a questionnaire to collect data on students' practices rather than direct observations. Since we collected data at the end of the first year, there is a potential for recall bias. Thus, a combined quantitative and a qualitative approach at regular periods during their first year would have gathered robust data on students' exposures to occupational hazards and their postexposure practices.

\section{Conclusions}

The study demonstrated that cut injuries, cadaver fluid contact with skin and eye splashes were commonly encountered during the first-year of dissections. Majority did not follow appropriate postexposure precautions which could minimize subsequent health problems. Some of the protocols practiced worldwide in laboratories which handle human tissues such as using lab coats were not strictly practiced in our laboratory. Creating awareness about the safety protocols among students is vital to minimize exposure to occupational hazards. We recommend conducting audits among students, academic staff and academic support staff on a regular basis.

\section{Acknowledgements}

The authors wish to thank Prof. VHW Dissanayake, Head, Chair and Senior Professor of Anatomy for the support given throughout the study.

Dr. Yasith Mathangasinghe, Lecturer, Faculty of Medicine, University of Colombo.

yasith@anat.cmb.ac.lk

Submitted by-March 2019

Accepted by-May 2019

References

1. Yaragalla, S. and A. Rajput, Identification of Fungal Growth from the Internal Organs of Preserved Human Cadavers. A m e rican J o u r n a 1 of Microbiological Research, 2017. 5(1): p. 25-27.

2. Ehdaivand, S., et al., Are biosafety practices in anatomical laboratories sufficient? A survey of practices and review of current guidelines. Human pathology, 2013. 44(6): p. 951-8. 
Mathangasinghe Y, Samaranayake UMJE, Perera MHS, Maddumaarachchi HSTM, Anthony DJ, Malalasekera AP - An audit on medical students' exposures to occupational hazards during cadaveric dissections

3. James, D.R., et al., Anatomy: the future teaching of undergraduates. Journal of Hospital Medicine, 2004. 65(11): p. 681-5.

4. Healing, T.D., P.N. Hoffman, and S.E. Young, The infection hazards of human cadavers. Communicable Disease Report CDR Review, 1995. 5(5): p. R61-8.

5. Harrington, J.M., Health and safety in medical laboratories. Bulletin of World Health Organization, 1982. 60(1): p. 9-16.

6. Demiryürek, D., A. Bayramoğlu, and Ş. Ustaçelebi, Infective agents in fixed human cadavers: a brief review and suggested guidelines. The Anatomical Record: An Official Publication of the American Association of Anatomists, 2002. 269(4): p. 194-197.

7. Chia, S.E., et al., Medical students' exposure to formaldehyde in a gross anatomy dissection laboratory. Journal of Americal College Health, 1992.41(3):p. 115-9.

8. Beane Freeman, L.E., et al., Mortality from lymphohematopoietic malignancies among workers in formaldehyde industries: the National Cancer Institute Cohort. Journal of the National Cancer Institute, 2009. 101(10): p. 751-761.

9. World Medical Association Declaration of Helsinki: ethical principles for medical research involving human subjects. Jama, 2013.310(20): p. 2191.
10. Palao, R., et al., Chemical burns: pathophysiology and treatment. Burns, 2010. 36(3): p. 295-304.

11. Cartotto, R.C., et al., Chemical burns. Canadian Journal of Surgery, 1996.39(3): p. 205-11.

12. Panel, B.B.R., Guidelines for safe work practices in human and animal medical diagnostic laboratories. Morbidity and Mortality Weekly Report, 2012.61.

13. St a ff, W. H.O. and W.H. Organization, Laboratory biosafety manual. 2004: World Health Organization.

14. Health, A.G.D.o., The Australian Immunisation Handbook. 2013: Department of Health and Ageing.

15. Salerno, R.M., J. Gaudioso, and B.H. Brodsky, Laboratory biosecurity handbook. 2007: Crc Press.

16. Safety Operating Procedure Biosafety Level 2 Laboratory. 4 ed. 2012: National University of Singapore.

17. Tarantola, A., et al., Occupational blood and body fluids exposures in health care workers: four-year surveillance from the Northern France network. American journal of infection control, 2003. 31(6): p. 357-63.

18. Chosewood, L.C., Biosafety in microbiological and biomedical laboratories. 2007: Diane Publishing. 
Mathangasinghe Y, Samaranayake UMJE, Perera MHS, Maddumaarachchi HSTM, Anthony DJ, Malalasekera AP - An audit on medical students' exposures to occupational hazards during cadaveric dissections

\section{Figures}

Figure 1: A scatterplot of showing correlations between frequency of skin contamination with cadaveric fluid (measured as the number of incidences per year) and the self-reported engagement in dissections (measured in a 10-point Likert scale; $1=$ never, $10=$ always). The shaded area represents the $95 \%$ confidence interval of the regression line.

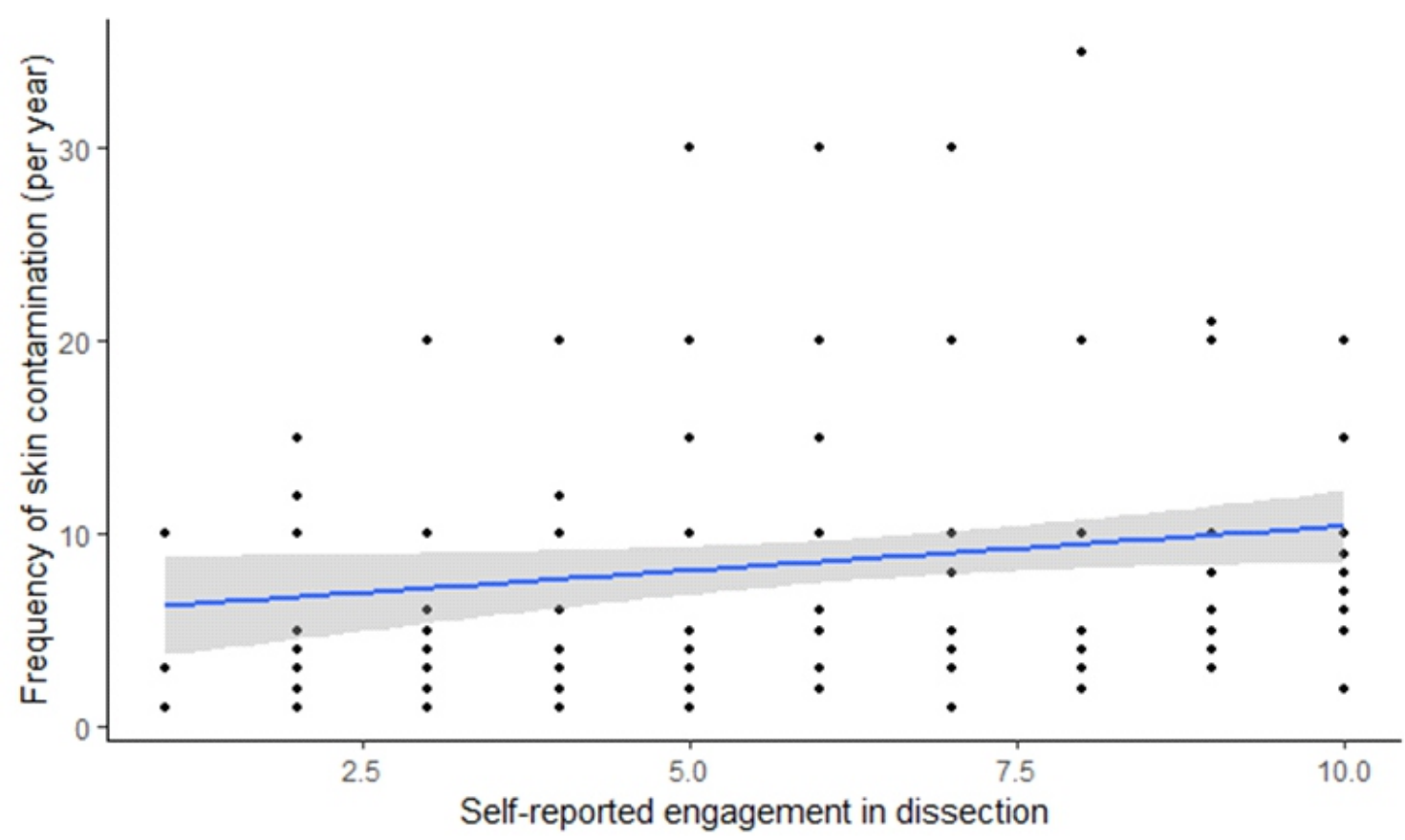

